\title{
Investigation of the initiation and propagation of cracks in the coated surface of spur gear: An application of irreversible cohesive zone model
}

\author{
Jiling Feng ${ }^{1, \mathrm{a}}$, Yi Qin ${ }^{2}$, and Hanshan Dong ${ }^{3}$ \\ ${ }^{1}$ Division of Mechanical Engineering, School of Engineering, Manchester Metropolitan University, \\ M1 5GD, UK \\ ${ }^{2}$ Centre for Precision Manufacturing, Dept. of Design, Manufacture \& Engineering Management, \\ The University of Strathclyde, Glasgow G1 1XJ, UK \\ ${ }^{3}$ Surface Engineering, University of Birmingham, Birmingham B15 2TT, UK
}

\begin{abstract}
Spur gears are the most common type of gears for industry, due to its simple structures and low costs of manufacture. Under the action of rolling contact fatigue, the main mode of the coated teeth failure is surface or subsurface originated micro-pitting, leading to the debonding of coating from substrate, fracture in the coating and even subsurface failure. Failure may originate from initiation of cracks, and its growth and propagation, however, basic failure mechanism is still not clear. In this paper, a comprehensive finite element modeling procedure including submodelling techniques and irreversible cohesive zone modelling techniques (CZM) is developed to investigate the failure mechanisms under the rolling contact fatigue loading. The details of the localized stresses distribution will be simulated using the submodelling technique. Irreversible CZM will be used to simulate the initiation and propagation of the cracks in the coated surface of the spur gears under the cyclic contact loading.
\end{abstract}

\section{Introduction}

Thin hard PVD (physical vapour deposition) coatings have been widely used in a variety of industrial areas including gear systems to enhance the hardness and wear resistance of contact surface. The reliable theoretical and numerical model for the coating surface design for engineering, however, is still not available. The requirements for surface engineering in specific applications, therefore, are usually depended on empirical formula and basis of experience. Apparently, a comprehensive computational model, allowing for the specific working conditions to be taken into account, is demanded, from the point of the coating design and analysis of view. Most recently, an innovative modelling strategy, which combined with the parameterized modelling, submodelling technique and cohesive zone model (CZM), was developed to determine the load bearing capacity for the coated products $[1,2]$. The advantage of this comprehensive modelling technique allows for many key factors, including geometry, material

\footnotetext{
${ }^{a}$ Corresponding author: j.feng@mmu .ac.uk
}

This is an Open Access article distributed under the terms of the Creative Commons Attribution License 4.0, which permits unrestricted use, distribution, and reproduction in any medium, provided the original work is properly cited. 

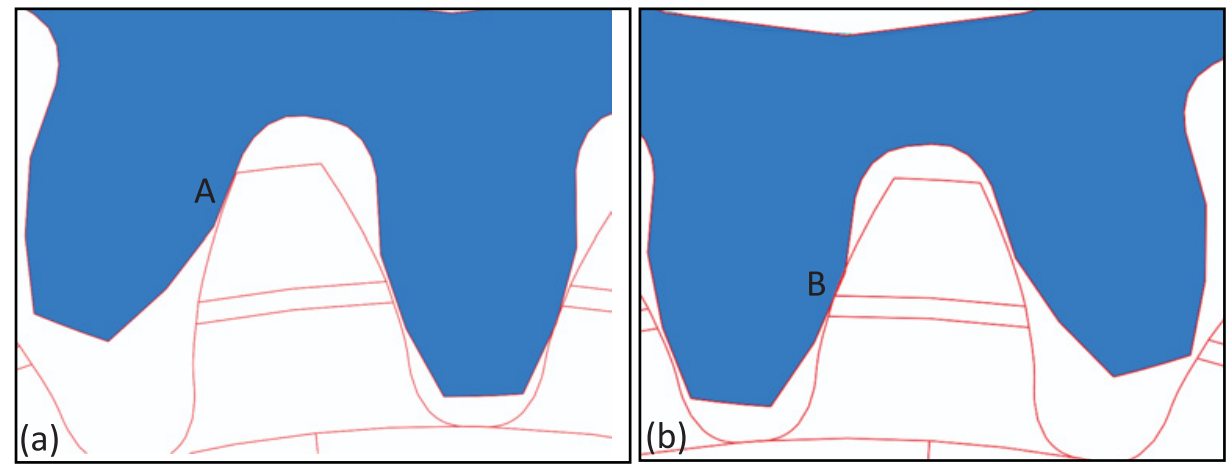

Figure 1. (a) Point A represents the location, where the pinion started to engage with wheel; (b) point B represents the location, where only one pair of teeth is subjected to load.

properties and pre-heated treatment to be considered for the coating design and its failure mechanism. This modelling strategy has been validated by a series of micro-indentation test and rolling contact test $[1,3]$. In this study, the function of this innovative modelling strategy is further developed by implementation of the irreversible cohesive law with unloading-reloading hysteresis, with which the failure mechanisms of the coated spur gear under the contact fatigue loading will be investigated.

\section{Localization of the damage on the tooth flank}

Based on the conventional standard procedures for gear design, pinions are more apt to pit than gears [4]. This is because the sliding motion of the pinion tends to pull metal away from the pitch line due to its role as a driver. In addition, the pinion has more cycles of operation than the gear wheel and therefore more apt to fail due to fatigue damage. In this study, investigation of damage behavior only focuses on the teeth flank of pinion.

In this paper, investigation of the damage behavior will be focused on two typical locations in the teeth flank of pinion (point A and point B in Fig. 1). The micro-pitting is assumed to occur at Point A due to its typical location. As is shown, at the point $\mathrm{A}$, the wheel tip touches pinion root and engagement begins. If the pinion has small number of teeth, the radius of curvature of involute profile at the root tends to reduce toward zero, which induces extremely high localized stresses, and hence giving rise to pitting away of the metal. At point B, where the load is only supported by one pair of teeth and highest contact stress is expected to occur, is considered as the area where the micro-pitting is easily to occur. Therefore, damage behavior such as localized contact stresses, plastic deformation and cracks due to micro-pitting at these two locations on the flank teeth of pinion will be investigated in this study.

\section{Cohesive zone model}

The concept of cohesive law specified the relationship of the traction and separation across the two virtual adjacent surfaces provides a phenomenological description for the progress damage and eventual fracture of the material [5]. Implementation of cohesive zone model in the between the continuum elements has been proved an efficient technique for the simulation of nucleation and propagation of cracks in the coating surface under monotonic loading [1]. However, under the fatigue loading condition, the deterioration of material properties and cohesive strength is expected to be related to the loading history. In this paper, CZM with unloading-reloading hysteresis will be implemented into the developed 
modelling technology, allowing for the fatigue failure mechanisms under the contact cyclic loading to be investigated.

\subsection{Cohesive-zone model under the monotonically loading}

An irreversible bilinear cohesive-zone modelling approach under the monotonically loading was incorporated with the other modelling techniques including parameterized modelling technique and submodelling technique to investigate the crack initiation within the coating layer. The bilinear cohesivezone law, characterised as the linear function of softening relationship of traction-separation of material degradation, can be written as:

$$
G_{c}=0.5 T_{\max } \delta_{c}
$$

where: $G_{c}$ is the critical energy-release rate governing the damage evolution and $T_{\max }$ indicates the maximum traction; and $\delta_{c}$ is the characteristic cohesive-zone length to which the separation reaches when the crack surfaces will be generated. A crack in the coating layer may be initiated when the separation reaches the critical value of, $\delta_{0}$, at which point the traction achieves its maximum value, $T_{\max }$. The parameters of cohesive properties, including the energy-release rate, are crucial to the accuracy of simulation of the crack within the coating and delaminating of bonding interface. In this study, it has been shown that the adhesive energy at atomistic level, can be calculated using the commercial software CASTEP, provides an alternative way to determine the value of the cohesive properties. It also provides an approach to link to micro- and nano-mechanical modelling. By means of cohesive-zone modelling approach, the critical load in terms of the initiation of the first crack is able to be predicted. More details about the bilinear cohesive constitutive law, the implementation and applications can be found in [1].

\subsection{Cohesive-zone model under cyclic loading}

The fatigue can be considered as one of the major causes which induce the failures in many coated surface-systems. Under a fatigue loading condition, the material deteriorates with the time and the prevailing cohesive strength must be related to the loading history. To develop a model that considers cyclic loading, an irreversible cohesive law with unloading-reloading hysteresis function, as shown in Fig. 2, is employed to simulate the mechanical response of the cohesive zone. This cohesive constitutive law, through introduction of damage variable, D, enables the degraded cohesive properties $(t-\varepsilon)$ [6]. The modified constitutive equations incorporating the damage variable, D, are written as,

$$
\left\{\begin{array}{l}
t_{n} \\
t_{s} \\
t_{t}
\end{array}\right\}=\left[\begin{array}{rrr}
K_{n n}(1-D) & & \\
& K_{s s}(1-D) & \\
& & K_{t t}(1-D)
\end{array}\right]\left\{\begin{array}{l}
\varepsilon_{n} \\
\varepsilon_{s} \\
\varepsilon_{t}
\end{array}\right\} .
$$

As mentioned above, the cohesive element has been implemented in the developed FE-based continue mechanics model with the commercial software ABAQUS 6.11. With the condition of cyclic loading, the modified cohesive properties as described above are modelled in the current model with use of the UMAT subroutine in ABAQUS. In this model the cohesive element possesses four nodes and two integration points, and the instance state of the cohesive element is determined by the modified cohesive constitutive equation.

\section{Methodology}

The modelling procedure in this study combines several modelling approaches, including the parameterized FE modelling, irreversible CZM and sub-modelling techniques. Parameterized modelling allows for materials, geometrical parameters as well as loading conditions to be described using 


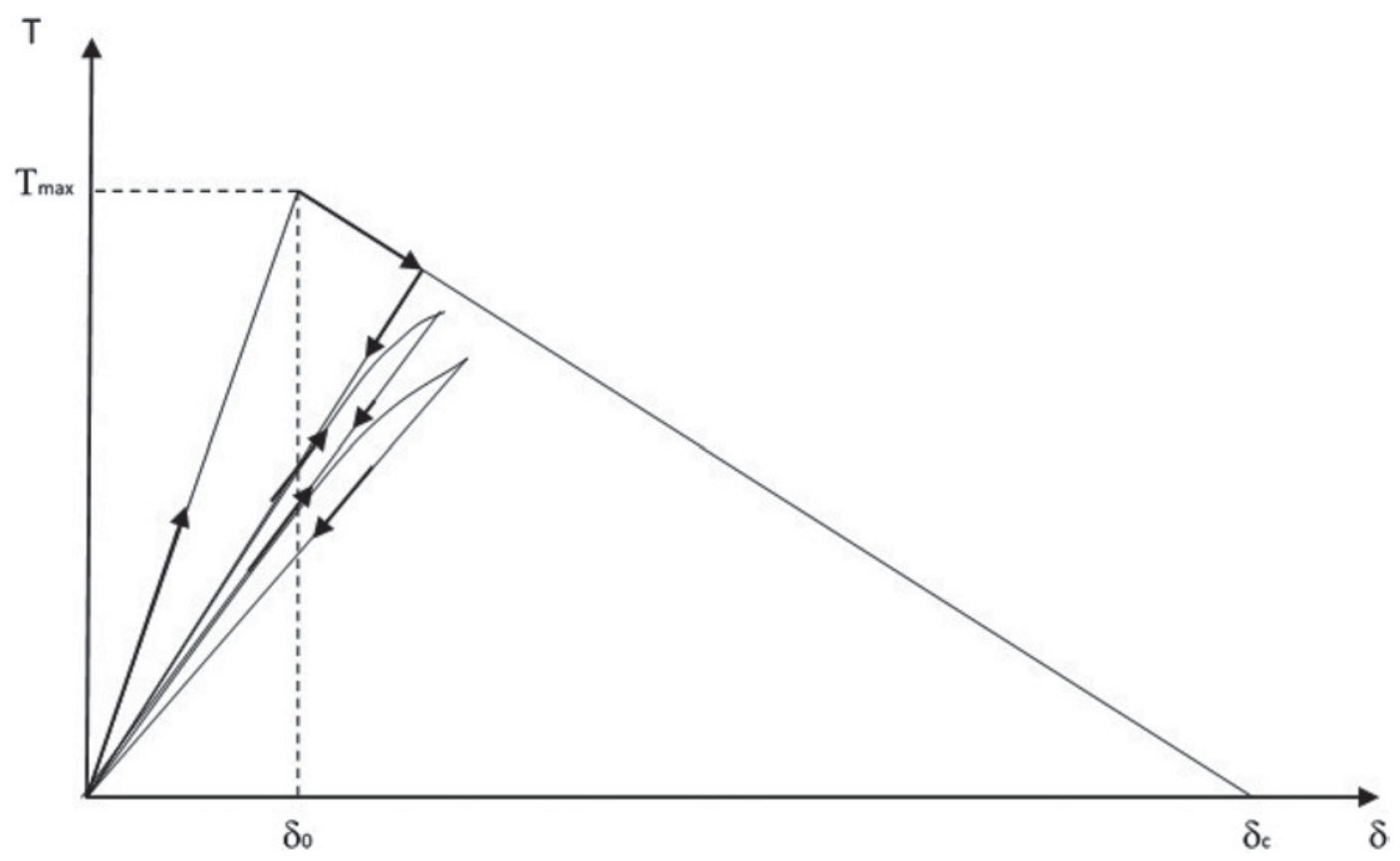

Figure 2. Cohesive Traction-Separation Law under Cyclic Loading.

parameters, enabling many key factors to be incorporated in the analysis as a whole. CZM enables the reproduction of the initiation and propagation of cracks in the coating-layer under complex gear/pinion contact loading conditions. The sub-modeling technique helps to scale down the geometry of the global model from the macro-scale to the micro/nano-scale, the sub-modeling technique is a powerful tool to understand the performance of coating structures and its failure mechanism in great details. Figure 3 shows the pair of spur gears as a FE model, where line contact between the pinion and the wheel was studied. In order to address the significant difference of dimensions between the wheel/pinion and coating thickness, a two-step analytical procedure was used: (1): Determining the contact conditions (pressure and friction) in the contact area using a global model; and (2): Generating a representative sub-model within the contact region so that coating performance can be investigated at the micron-scale level. It is down to the micro scale level that cohesive elements were introduced in order to predict the performance of coating system and its damage micro mechanism.

\section{Case study}

Crack initiation and propagation analysis had been carried out for a 42CrMo4 spur gear with deposited multilayer TiN/Cr coatings. Two different surface systems were investigated in this study and they are defined as the following: (1) Model A: a model PVD TiN/CrN coating on a T-condition 42CrMo4; (2) Model B: a model PVD TiN/CrN coating on a nitride 42CrMo4 substrate.

The spur gears were manufactured in $42 \mathrm{CrMo} 4$ steel and then the gears were super-finished. For the model A, the steel substrate of spur gear was directly coated with TiN/CrN coating. For the model B, prior to the coating deposition, the part of steel substrates was subjected to active-screen plasma nitriding, whereas the other part was in the simple quenched-and-tempered state. This type of surface system was referred as nitrided $42 \mathrm{CrMo} 4$ substrate. Coating deposition was performed with 


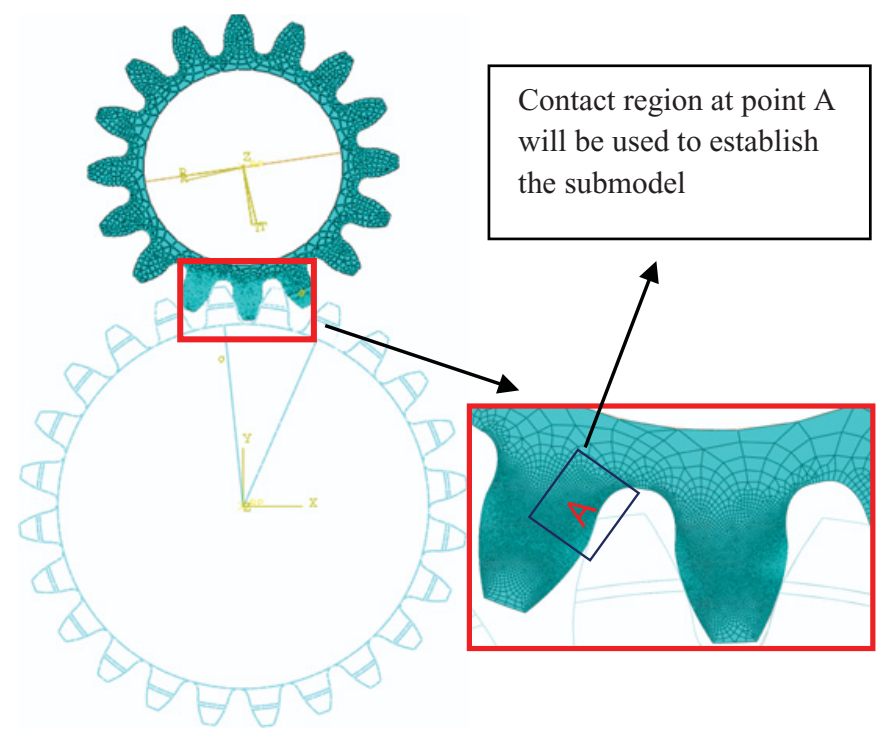

Figure 3. Engagement of the pinion and wheel. The gear starts to touch root of tooth of pinion (point A) and the engagement starts. The submodel, representing the contact conditions around the point A, is established to investigate the details of the localized contact stresses and crack propagation at this area.

Table 1. Material properties of coating and substrate.

\begin{tabular}{|c|c|c|c|}
\hline Materials & Thickness (micron) & Hardness (HV) & Modulus (GPa) \\
\hline CrN/TiN & 2 & 1680 & 240 \\
\hline Substrate & - & 300 & 120 \\
\hline
\end{tabular}

an industrial Cathodic Arc Evaporation PVD system. The configurations of the two types of gears are summarizes as:

- Model A: a model PVD TiN/CrN coating on a T-condition 42CrMo4

- Model B: a model PVD TiN/CrN coating on a nitride 42CrMo4 substrate.

In both FE global and the sub-model, the coating and substrate are characterized as homogenous elastic plastic material of linear hardening behavior. The depth dependent hardness of the hardened layer was converted into yield strength, which is obtained by the least square fitting-technique and implemented with the field method.

The material properties for both substrate and coating are measured in Department of Material Engineering in Birmingham University and displayed in Table 1.

\section{Discussion and conclusions}

The results from the FE model in this study was compared with the experimental results obtained from DGMK-FZG (Deutsche Wissenschaftliche Gesellschaft für Erdöl, Erdgas und Kohle, Forschungsstelle für Zahnräder und Getriebebau der TU München) short micro-pitting test. In such tests, different levels of load (Torque) were applied on the pinion/gear system. During experiment, the critical loads were defined as the load at which surface cracks nucleate, where cracks were detected with scanning electron microscopy (SEM) by in-situ observations (Fig. 4). 

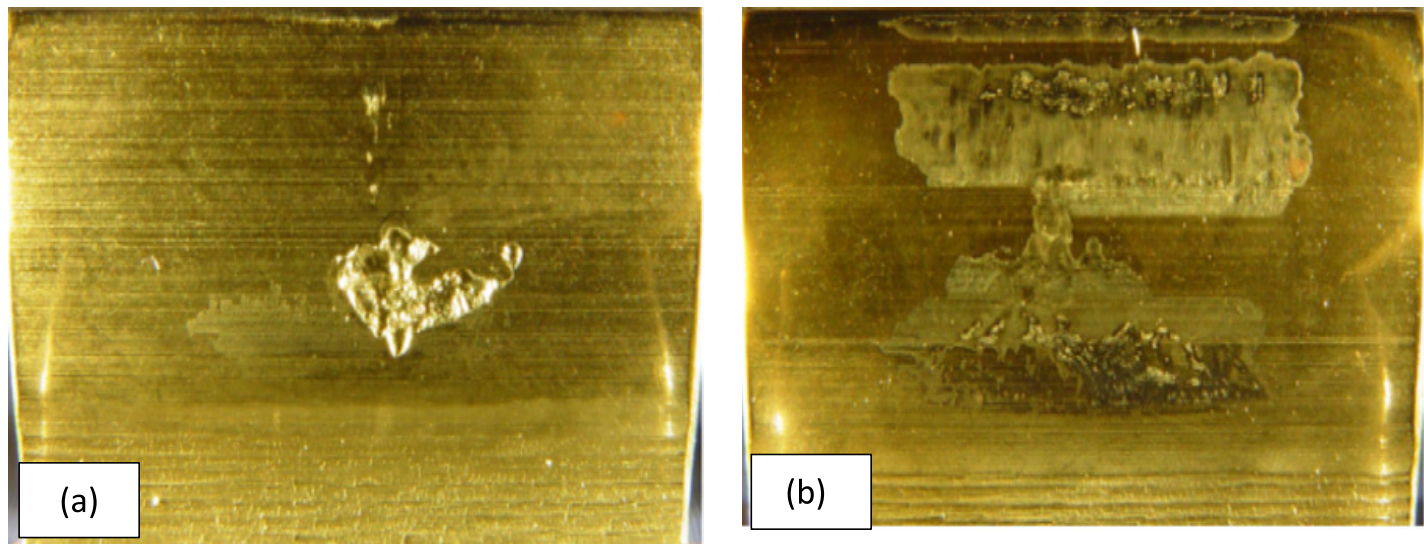

Figure 4. Photography of the fail test for the coated tooth Model A and Model B. (a) Model fails at key stage loading 6; (b) model B fails at key stage loading 8.
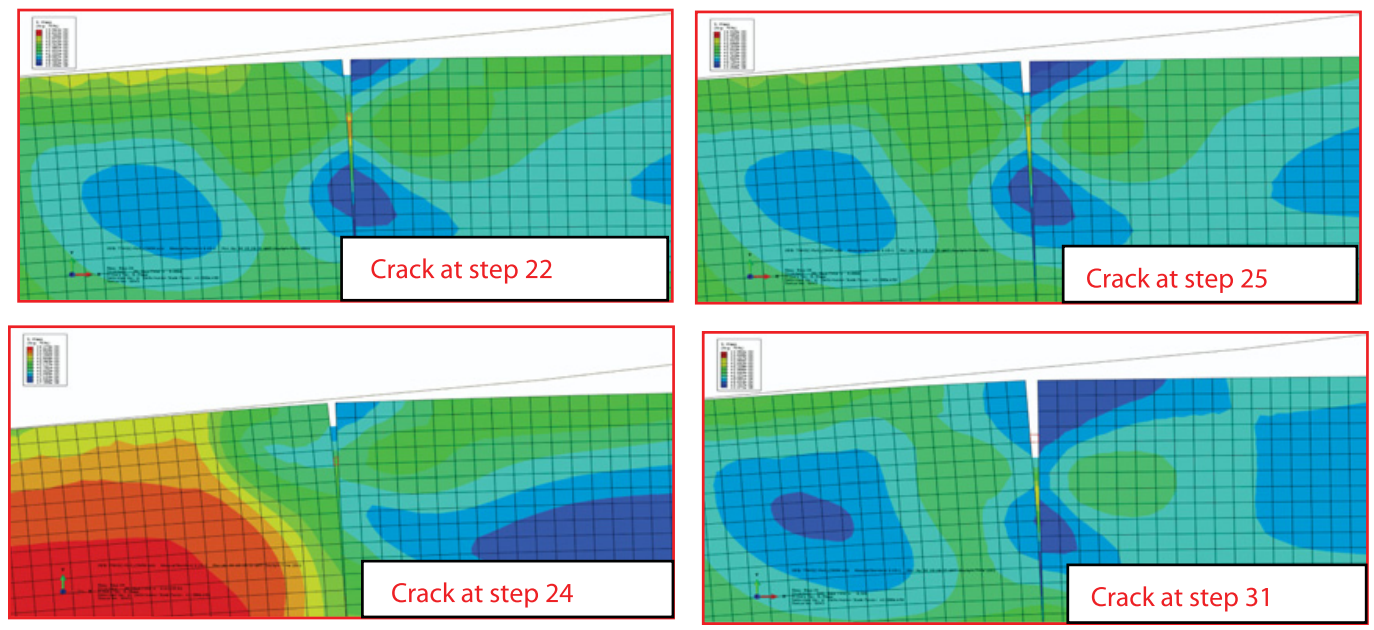

Figure 5. Crack propagation with the increase of loading cycles.

Similar to the test procedure, different levels of load (Toque) were also applied on the global model (pinion/gear system). The pressure distribution at the contact region (point A and point B) from the global model was then input to sub-model as the boundary conditions. The load bearing capability was defined as the load at which when the 1st crack was initiated. The critical load predicted through FE simulation are in agreement with those observed from SEM images. The results also shown that the material strength degraded with increase of loading cycles, which led to the rapidly damages of coated surface once the crack was nucleated. With the increase of contact loading cycles, the cracks propagate rapidly to the depth of the coating, which will eventually result in the debonding of the coating from the substrate. The crack propagation in the surface of coating as the loading cycles increases is shown in Fig. 5.

As is seen in Table 2, average pressure at point A was greater than that at point B for both types of gears although the normal force acted in this region was much smaller than in the region around point $\mathrm{B}$. The reason for that was the small radius of curvature of involute profile at the root leading to the high localized stresses and eventually giving rise to nucleation of the crack in this area. It was found from 
ICNFT 2015

Table 2. Critical loads for two types of surface systems.

\begin{tabular}{|l|c|c|c|c|c|}
\hline \multicolumn{2}{|l|}{$\begin{array}{l}\text { Critical load for crack } \\
\text { initiation }\end{array}$} & $\begin{array}{l}\text { Maximum } \\
\text { pressure [MPa] }\end{array}$ & $\begin{array}{l}\text { Average } \\
\text { pressure [MPa] }\end{array}$ & Normal force [N] & $\begin{array}{l}\text { Torque } \\
{[\mathbf{N m}]}\end{array}$ \\
\hline \multirow{2}{*}{ Model A } & Point A & 1438 & 1321 & 1644 & \multirow{2}{*}{170} \\
\cline { 2 - 5 } & Point B & 1534 & 1279 & 5014 & \multirow{2}{*}{358} \\
\hline \multirow{2}{*}{ Model B } & Point A & 3768 & 3331 & 4883 & 10588 \\
\cline { 2 - 6 } & Point B & 3245 & 2918 & & \\
\hline
\end{tabular}

both experimental and computational results that pre-heated treatment with plasma nitriding improved the surface properties dramatically, where the critical load associated with initiation of first crack for Model B system is twice time than that for Model A system. The greater critical load with the TiN/CrN on nitrided $42 \mathrm{CrMo} 4$ may be due to the improved properties of the hardened case.

This research was support by the FP7 project "Multiscale Modelling for Multilayered Surface Systems (M3-2S)", Grant No. CP-FP213600-2 M3-2S.

\section{References}

[1] J. Feng, Y. Qin, Q. Zeng, E. Alamandoz, G. Fuente, H. Dong, R. Raghavan, J. Michler, J. Multiscale Model. 1, 1 (2011)

[2] J. Feng, Y. Qin, Appl. Mech. Mater. 446, 491 (2014)

[3] J. Feng, Y. Qin, M. Lorusso, D. Ugues, in Proceedings of the 14th International Conference on Metal Forming "Metal Forming 2012", edited by J. Kusiak, J. Majta, D. Szeliga (Wiley-VCH Verlag GmbH \& Co. KGaA, Krakow, 2012), p. 1059

[4] D.W. Dudley, Handbook of Practical Gear Design (Dudley Engineering Co. McGraw-Hill Book Company, US, 1984)

[5] X.P. Xu, A. Needleman, J. Mech. Phys. Solids 42, 1397 (1994)

[6] A. Abdul-Baqi, P.J.G. Schreurs, M.G.D. Geers, Int. J. Solid Struct. 42, 927 (2005) 